\title{
Fast radio bursts: the last sign of supramassive neutron stars
}

\author{
Heino Falcke $e^{1,2,3}$ and Luciano Rezzolla $a^{4,5}$ \\ ${ }^{1}$ Department of Astrophysics, Institute for Mathematics, Astrophysics and Particle Physics, Radboud University Nijmegen, \\ PO Box 9010, 6500 GL Nijmegen, The Netherlands \\ e-mail: H.Falcke@astro.ru.nl \\ 2 ASTRON, Oude Hoogeveensedijk 4, 7991 PD Dwingeloo, The Netherlands \\ 3 Max-Planck-Institut für Radioastronomie, auf dem Hügel 69, 53121 Bonn, Germany \\ 4 Max-Planck-Institut für Gravitationsphysik, Albert-Einstein-Institut, 14476 Potsdam, Germany \\ 5 Institut für Theoretische Physik, 60438 Frankfurt am Main, Germany
}

Received 30 May 2013 / Accepted 20 January 2014

\begin{abstract}
Context. Several fast radio bursts have been discovered recently, showing a bright, highly dispersed millisecond radio pulse. The pulses do not repeat and are not associated with a known pulsar or gamma-ray burst. The high dispersion suggests sources at cosmological distances, hence implying an extremely high radio luminosity, far larger than the power of single pulses from a pulsar. Aims. We suggest that a fast radio burst represents the final signal of a supramassive rotating neutron star that collapses to a black hole due to magnetic braking. The neutron star is initially above the critical mass for non-rotating models and is supported by rapid rotation. As magnetic braking constantly reduces the spin, the neutron star will suddenly collapse to a black hole several thousand to million years after its birth.

Methods. We discuss several formation scenarios for supramassive neutron stars and estimate the possible observational signatures making use of the results of recent numerical general-relativistic calculations.

Results. While the collapse will hide the stellar surface behind an event horizon, the magnetic-field lines will snap violently. This can turn an almost ordinary pulsar into a bright radio "blitzar": accelerated electrons from the travelling magnetic shock dissipate a significant fraction of the magnetosphere and produce a massive radio burst that is observable out to $z>0.7$. Only a few per cent of the neutron stars need to be supramassive in order to explain the observed rate.

Conclusions. We suggest the intriguing possibility that fast radio bursts might trace the solitary and almost silent formation of stellar mass black holes at high redshifts. These bursts could be an electromagnetic complement to gravitational-wave emission and reveal a new formation and evolutionary channel for black holes and neutron stars that are not seen as gamma-ray bursts. If supramassive neutron stars are formed at birth and not by accretion, radio observations of these bursts could trace the core-collapse supernova rate throughout the universe.
\end{abstract}

Key words. pulsars: general - magnetic fields - gravitation - radiation mechanisms: non-thermal - gamma-ray burst: general stars: neutron

\section{Introduction}

Recently, a number of isolated fast radio bursts (FRBs) have been discovered that last for only a millisecond and which may come from cosmological distances (Lorimer et al. 2007; Thornton et al. 2013). What could possibly produce such a bright emission in such a short time? A natural explanation may be gravitational collapse involving neutron stars (NSs) or stellar mass black holes (BHs). Typically, the formation of NSs and $\mathrm{BHs}$ are associated with some rather energetic observational signatures across all wavelengths, such as a supernova $(\mathrm{SN})$ or a gamma-ray burst (GRBs). The latter are short-term flares of $\mathrm{X}$-ray and gamma-ray emission, lasting only a fraction of seconds to tens of seconds, sometimes with an extended afterglow. The total energy radiated in a GRB is $\sim 10^{48-50} \mathrm{erg} \mathrm{s}^{-1}$ and the bright emission was explained initially in a fireball model (Cavallo \& Rees 1978; Paczynski 1986; Eichler et al. 1989), where a significant fraction of the energy is thermalized in an optically thick outflow eventually radiated in the form of highenergy emission (see Nakar 2007; Lee \& Ramirez-Ruiz 2007, for recent reviews).

Short GRBs, of duration less than $2 \mathrm{~s}$, are thought to be associated with NS-NS mergers and not to trace well star formation
(Gehrels et al. 2005). Their average timescale is around $50 \mathrm{~ms}$ (Nakar 2007) with some spread. Long GRBs, with a duration longer than $2 \mathrm{~s}$, may be associated with the $\mathrm{SN}$ of a massive star, thereby well tracing cosmic star formation (Woosley \& Bloom 2006). For the latter scenario, the GRB is suggested to be due to a plasma jet that propagates through the dense outer layers of the exploding star (Woosley 1993). Hence, baryon loading and particle acceleration in internal or external shocks play an important role in the observational appearance of GRBs. However, do all forms of collapse lead to such bright observational signatures?

We here discuss the collapse of an isolated and magnetized supramassive rotating neutron star (SURON) to a BH in a rarefied environment. Such a collapse would be inevitable if a rapidly spinning NS was formed above the critical mass for a non-rotating NS. Over time, magnetic braking would clear out the immediate environment of the star and slow it down. With a significant delay after the creation of the NS in a supernova implosion, the SURON will eventually collapse almost instantly. The formation of an event horizon over the free-fall timescale, i.e. $<1 \mathrm{~ms}$, during the collapse would immediately hide most of the matter and radiation apart from the SURON's magnetosphere. Instead, the magnetosphere will experience a violent disruption leading to a strong magnetic shock wave travelling 
outwards near the speed of light and producing radio emission. Hence, the observational signatures of such a system would be quite different from those of short or long GRBs and more akin to that of pulsar emission.

The collapse of a SURON near break-up spin has already been considered by Vietri \& Stella (1998), who suggested that, while the collapse itself is "silent", accretion from a torus, consisting of the mass shed from the stellar equator, onto the newly formed BH could power a wind that is detectable as an afterglow. The "silence" in a NS collapse comes from the fact that the $p \mathrm{~d} V$-work done by the contracting star which could heat it up, cannot escape via conduction or neutrinos because of the very short timescales involved and thus ends up in the $\mathrm{BH}$.

We now know, through general-relativistic simulations of the collapse of SURONs near break-up, that such a torus does not seem to form (see Fig. 16 of Baiotti et al. 2005). At the same time, recent general-relativistic simulations in resistive magnetohydrodynamic (MHD) also tell us that the collapse of magnetized NSs is accompanied by a strong electromagnetic emission (Lehner et al. 2012; Dionysopoulou et al. 2013). Hence, a collapsing SURON is not entirely "silent" and, as we will discuss, by simply using the power stored in the magnetosphere, a detectable prompt signature should be produced in the radio regime.

In fact, the recently discovered FRBs may make this scenario an intriguing possibility. In 2007 Lorimer et al. discovered a single bright and highly dispersed radio flash in archival pulsar survey data of the Parkes telescope that was not associated with any known pulsar or GRB. In the meantime, more of these events have been found (Keane et al. 2012; Thornton et al. 2013). While a terrestrial origin of these radio signals can not be fully excluded (Burke-Spolaor et al. 2011) and the bursts have not been confirmed by other telescopes yet (e.g., Bower et al. 2011; Siemion et al. 2012), an origin of these one-off radio bursts at cosmological distances now seems a viable interpretation (Thornton et al. 2013; Lorimer et al. 2013).

Theoretical interpretations of the Lorimer burst have been rare until now and no canonical picture has emerged yet (Thornton et al. 2013). A connection with short hard GRBs and merging NSs has been proposed (Pshirkov \& Postnov 2010; Lyutikov 2013), as well as supernova explosions in a binary system impacting a NS magnetosphere (Egorov \& Postnov 2009), reconnection in the magnetosphere of NSs (Somov 2011), or even superconducting cosmic strings (Cai et al. 2012) and the evaporation of BHs in the presence of extra spatial dimensions (Kavic et al. 2008). In light of the new observations it may be worth revisiting these scenarios ${ }^{1}$.

The basic properties of the six currently known radio bursts can be summarized as follows: they are short with timescales $\Delta t \lesssim 1 \mathrm{~ms}$; the radio fluxes are typically around $1 \mathrm{Jy}$ at $\mathrm{GHz}$ frequencies, with the brightest and closest one reaching over $30 \mathrm{Jy}$; no associated GRBs have been seen at the time of the bursts; the rate is about 0.25 per square degree per day; the radio burst is dispersed, with shorter wavelengths preceding longer ones, following a $\Delta t \propto \lambda^{2}$ law. The dispersion is similar to what is seen in pulses from Galactic pulsars due to free electrons in the interstellar medium. However, the derived dispersion measures (DM), in the range of a few hundred to thousand $\mathrm{pc} \mathrm{cm}^{-2}$, far exceed the Galactic DM in those directions. Hence, dispersion due to the intergalactic medium has been suggested, which provides distance

\footnotetext{
1 Since the submission of this paper, other interpretations and models have been put forth to explain FRBs; see, e.g., Totani (2013); Katz (2013); Kashiyama et al. (2013); Loeb et al. (2014); Luan (2014).
}

estimates of several Gpc and redshifts in the range $z=0.3-1$. In two cases, there is also evidence for a frequency-dependent scattering tail as expected for bursts passing through a turbulent ionized medium (Lorimer et al. 2007; Thornton et al. 2013).

The distribution of the few bursts would be consistent with the cosmological star formation or core-collapse SN rate. The apparent isotropic luminosity of these bursts at a mean redshift of $z=0.7$ and luminosity distance $D_{1} \sim 4.3 \mathrm{Gpc}$ (using $H_{0}=$ $79.4 \mathrm{~km} \mathrm{~s}^{-1} \mathrm{Mpc}^{-1}, \Omega_{\mathrm{m}}=0.27, \Omega_{\mathrm{vac}}=0.73$ ), for an observed spectrum $S_{v} \propto v^{\alpha}$, would be

$$
L=3 \times 10^{43}\left(\frac{v}{1.4 \mathrm{GHz}}\right)^{1+\alpha}\left(\frac{S_{v}}{1 \mathrm{Jy}}\right)\left(\frac{D_{1}}{11 \mathrm{Gpc}}\right)^{2} \mathrm{erg} \mathrm{s}^{-1} .
$$

This luminosity is more than nine orders of magnitude brighter than a giant kJy flare from the Crab pulsar. On the other hand, integrated over $1 \mathrm{~ms}$ this yields $3 \times 10^{40} \mathrm{erg}$, which is only a tiny fraction of a SN energy and much less than a typical GRB. Of course, the total luminosity could be somewhat higher if the spectrum were flat $(\alpha \simeq 0$ ) and would extend to higher frequencies.

Finally, we point out that the observed timescale of $\lesssim 1 \mathrm{~ms}$, together with the high luminosity and non-repetitive nature is very constraining. This is much shorter than that of $\mathrm{SNe}$, long and short GRBs, or of merging NS binaries. This points towards the shortest timescale available for compact objects, namely, the free-fall timescale of NSs. Also, the lack of GRB signatures and the appearance of high-brightness temperature emission, implying coherent emission processes, is very reminiscent of emission mechanisms of pulsars. Hence, in the following we propose a scenario that is able to address all these issues.

\section{Braking-induced collapse of a supramassive NS}

The basic scenario we consider here assumes an initial state of a magnetized NS with (gravitational) mass $M$ and spin frequency $\Omega=2 \pi \tau^{-1}$, where $M_{\max }(\Omega)<M<M_{\max }\left(\Omega_{\mathrm{K}}\right)$ and $\tau$ is the spin period. Here $M_{\max }(\Omega)$ is the maximum mass above which a NS is unstable to a collapse to a $\mathrm{BH}$ and $\Omega_{\mathrm{K}}$ is the maximum spin a NS can have, sometimes called Keplerian or break-up spin. In the absence of rotation, $M_{\max }(0)$ would be the equivalent of the Chandrasekhar limit for NSs. Its exact value depends on the still unknown equation of state (EOS) of nuclear matter, but NSs with masses larger than $M_{\max }(0)$ can still be stable if they are supported by centrifugal forces. If we assume the NS is spinning at a fraction $f$ of the break-up spin, i.e., $\Omega=f \Omega_{\mathrm{K}}$, then the resulting period is

$\tau_{\text {rot }}=2 \pi f^{-1} \sqrt{\frac{R^{3}}{G M}} \simeq 3.8 f_{0.1}^{-1} r_{10}^{3 / 2} m_{2}^{-1 / 2} \mathrm{~ms}$,

where $M=m_{2} 2.3 M_{\odot}$ is the mass of the NS, $R=r_{10} 10 \mathrm{~km}$ its radius, $G$ the gravitational constant, and $f_{0.1}=0.1 f$. Standard magnetic dipole radiation would then lead to a braking of the system on a timescale

$\tau_{\text {braking }}=\frac{6 c^{3} M \sin ^{-2}\left(\alpha_{\mathrm{B}}\right)}{5 B^{2} R^{4} \Omega^{2}} \simeq 3.1 f_{0.1}^{-2} r_{10}^{-1} b_{12}^{-2} \mathrm{kyr}$,

where $B=b_{12} 10^{12} \mathrm{G}$ is the magnetic field, $c$ is the speed of light, and $\alpha_{B} \simeq 45^{\circ}$ is the magnetic pitch angle. Hence, similar to highly magnetized pulsars (Duncan \& Thompson 1992) the source, if highly spinning, would slow down significantly within a few hundred to a few thousand years. If the NS is supramassive 


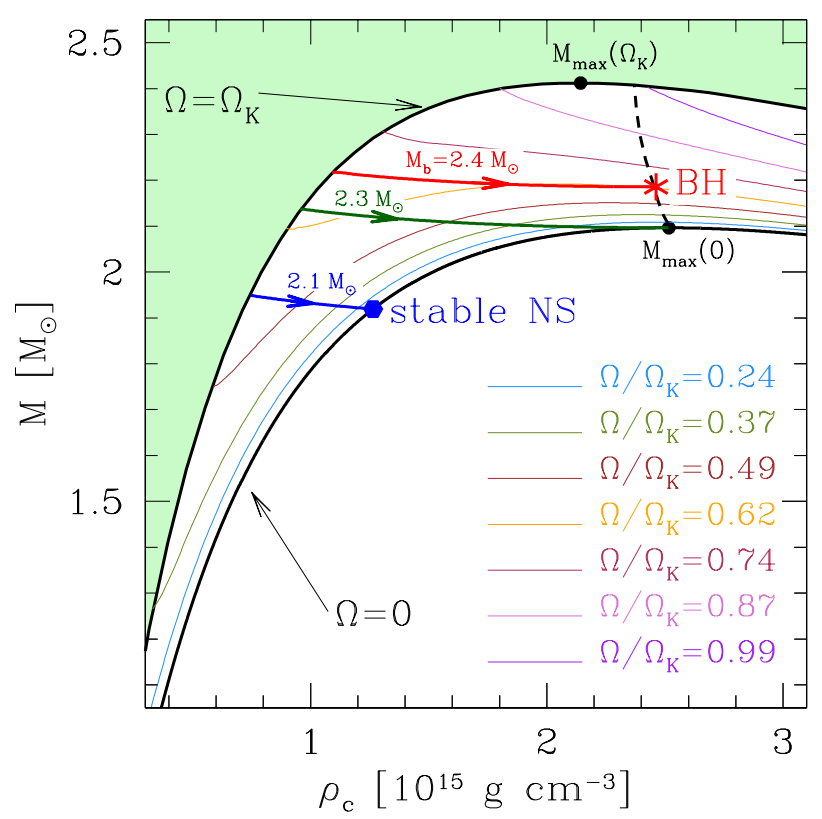

Fig. 1. Gravitational mass versus central rest-mass density for NS models with different spins. The green shaded area is the region where no equilibrium models are possible because of excessive spin. The black solid lines are sequences of models that are non-rotating, $\Omega=0$, or rotating at break-up, $\Omega=\Omega_{\mathrm{K}}$, respectively (see text for more details). The arrows indicate example tracks of NSs with baryonic masses of 2.1,2.3, and $2.4 M_{\odot}$ as they slow down due to magnetic braking.

it will collapse to a $\mathrm{BH}$ and hence disappear from the general pulsar population.

Calculating the stability of rotating NSs is far from trivial. We here make use of fully general-relativistic numerical calculations where the star is modeled as a uniformly rotating polytrope with index $\Gamma=2$ (Takami et al. 2011) and a polytropic constant chosen so that $M_{\max }(0)=2.1 M_{\odot}$ to match recent observations of high-mass NSs (Antoniadis et al. 2013). Although simplified, our EOS provides here a simple and overall realistic reference.

Figure 1 illustrates the results of these calculations in a diagram showing the gravitational mass $M$, versus the central restmass density $\rho_{\mathrm{c}}$. The black solid lines are sequences of models that are non-rotating, $\Omega=0$, or rotating at break-up, $\Omega=\Omega_{\mathrm{K}}$, respectively. Hence, the green shaded area indicates the region where no equilibrium models are possible because they would be past the break-up limit. Shown as coloured lines are sequences of constant angular frequency normalized to the maximum possible spin frequency, i.e., $\Omega / \Omega_{\mathrm{K}}$. We also show three sequences of constant baryon mass $M_{\mathrm{b}}$, which can therefore be interpreted as evolutionary tracks of a NS as it spins down during its life. It is then easy to realize that, for instance, a SURON with $M_{\mathrm{b}}=2.4 M_{\odot}$ (red solid line) could be produced near the breakup limit (but also at smaller rotation rates) and then spin down while maintaining its baryon mass. This corresponds to a motion to the right in Fig. 1, during which the NS reduces its gravitational mass (it contracts because of the decreased spin). This motion terminates around the stability line, the locus of the maxima of sequences of constant angular momentum, beyond which the star collapses to a Kerr BH (shown as a black dashed line). Similarly, a SURON with $M_{\mathrm{b}}=2.3 M_{\odot}$ (green solid line) would also spin down, but now to a zero spin frequency, when it reaches $M_{\max }(0)$. At that point it will have become a spherical star and any perturbation will induce its collapse to a Schwarzschild $\mathrm{BH}$. Finally, a normal NS with $M_{\mathrm{b}}=2.1 M_{\odot}$ (blue solid line) would also spin down to a zero frequency, but end up on the stable branch of equilibrium models, remaining there ever after.

In essence, for our representative EOS any spinning NS with a $M>2.1 M_{\odot}\left(M_{\mathrm{b}}>2.3 M_{\odot}\right)$, will eventually collapse to a $\mathrm{BH}$. The lifetime of the SURON will be of the order $\sim \tau_{\text {braking }}$ and since this depends on $f^{-2}$, it follows from Fig. 1 that NSs which are just a few percent above $M_{\max }(0)$ have the longest lifetimes and can collapse millions of years after their formation. On the other hand, a NS with $M_{\mathrm{b}} \simeq 2.4 M_{\odot}\left(M \simeq 2.3 M_{\odot}\right)$ has a decay timescale of about 3000 years. Hence, SURONs are not necessarily highly spinning.

All these timescales are long enough for the initial SN to have faded away by the time of collapse. Moreover, these timescales are also long enough that the baryonic pollution of the NS surroundings will have been cleared out, e.g., through ejection, fall-back, or magnetic winds, such that a magnetosphere is established. Clear evidence for this is given by the Crab pulsar, which is pulsating already $10^{3}$ years after its formation.

We also note that neutrino cooling and the associated mass loss is important only during the first 10-20 s after the formation of the proto-NS (12 s were measured for SN1987A), which can be as large as $10 \%$ of the mass. Only a few days later, however, the temperature has already decreased to $\sim 0.1 \mathrm{MeV} \sim 10^{9} \mathrm{~K}$, so that even if the star cooled down to a zero temperature it would lose at most an equivalent mass $\sim 0.1 \mathrm{MeV} \times 10^{57}$ nucleons $\simeq$ $10^{50} \mathrm{erg} \simeq 10^{-4} M$. In other words, while neutrino cooling can lead to observational signatures also thousands of years after the NS formation, see, e.g., Page et al. (2011), it soon becomes irrelevant in determining the equilibrium properties of the SURON.

Hence, we argue that, if SURONs are formed, they will have to collapse eventually and the collapse will take place in a clean environment that is rather different from that typically invoked for GRBs.

The rates estimated by Thornton et al. (2013) for FRBs are $\sim 0.25 \mathrm{deg}^{-2} \mathrm{day}^{-1}$. This we can compare to the observed rate of core collapse SN, e.g., Dahlen et al. (2004), which is within a factor of two from the core-collapse $\mathrm{SN}$ rate expected from the star formation rate (Horiuchi et al. 2011).

Extrapolating this to $z=1$, using the fitting function provided in Horiuchi et al. (2011), we arrive at a rate of $8 \mathrm{SNe} \mathrm{deg}^{-2} \mathrm{day}^{-1}$. Hence, we need only about $3 \%$ of the massive stars to undergo a collapse as we envisage here to produce the FRBs. Given that the fraction of rapidly-rotating massive stars could be up to $20 \%$, mainly due to binary interaction (de Mink et al. 2013), this suggestion does not seem unreasonable. Rotation also plays an important role in models for progenitors of long GRBs (Yoon et al. 2006), where an almost critically rotating iron core is needed. Spin-down of the progenitor, coupled to stellar mass loss (Langer 1998), might then introduce some metallicity bias, such that the SURON formation rate increases with lower metallicity.

The main uncertainty, however, is how a SN with a rapidly rotating progenitor proceeds to the NS stage. In principle, rapidly spinning NSs with high magnetic fields can be formed (Fryer \& Warren 2004) in a SN, but the magnetic field could also cause the NS to slow down already during the formation process. Still, it is not inconceivable that at least SURONS which are a few percent above critical mass and hence only need relatively modest spins can be formed. Therefore, the important question to address in the future is whether the rate of SURONs formed in direct $\mathrm{SN}$ explosions can ineed be on the few percent level.

Given that binary interactions play such an important role, it is possible to consider also an alternative formation channel, where a slowly rotating NS gains mass and spin through 
accretion from its companion in ultra-compact X-ray binaries (van Haaften et al. 2013) as in the standard scenario (Bhattacharya \& van den Heuvel 1991) for ms-pulsars (MSPs).

In fact, the so-called "black widow" systems can show rather massive NSs, with masses up to $2 M_{\odot}$ (Demorest et al. 2010), which might have already been born massive before accretion and spin-up (Tauris et al. 2011). The problem here is that spinup seems to suppress the magnetic field and it is not clear whether accretion-induced spin-up can preserve the strong magnetic fields needed for the emission. If SURONs were produced in this way, the number of detectable ones could be small because of the fast spin-down and short lifetime for the most massive stars.

Lastly, it is possible to consider a formation scenario that was originally proposed to explain MSPs, where a NS and a white dwarf merge (van den Heuvel \& Bonsema 1984). This too might lead to a rapidly spinning, highly magnetized, and "overweight" NS. Any scenario involving pulsar recycling rather than a direct formation, however, would not follow the high-mass star formation rate and hence can be observationally discriminated with more bursts to come.

\section{Emission from the collapse}

Next we estimate the observational signatures of the collapse of a SURON. Given that pulsar-emission mechanisms are notoriously difficult to calculate, we concentrate on an order-ofmagnitude description and a discussion of the basic mechanisms. The relevant timescale for the collapse is the free-fall timescale, $\tau_{\mathrm{ff}}=0.04 r_{10}^{3 / 2} m_{2}^{-1 / 2} \mathrm{~ms}$ (Baiotti et al. 2005; Baumgarte \& Shapiro 2003; Giacomazzo et al. 2011; Lehner et al. 2012).

Within a few $\tau_{\mathrm{ff}}$, the crust of the NS, that is crucial for thermal X-ray emission, will be covered by the emerging event horizon. For example, the time from the first appearance of the event horizon near the center of the NS until the NS surface is entirely covered by the event horizon can be as short as $0.15 \mathrm{~ms}$ for a slowly rotating star with dimensionless spin $J / M^{2} \sim 0.2$ (Baiotti et al. 2005, Fig. 16, left panel). This is a situation we could envisage for our SURONs. For a star near break-up and with $J / M^{2} \sim 0.54$, this duration increases to $0.35 \mathrm{~ms}$ (Baiotti et al. 2005, Fig. 16, right panel $)^{2}$. This is also the timescale over which the largest changes in stellar structure take place and most potential energy is liberated. Hence, there is a marked difference with respect to binary NS mergers and short GRBs, whose characteristic timescales are at least one order of magnitude longer.

Given the short timescale of the collapse, heat generated in the collapsing NS cannot be transported to the surface and radiated away efficiently, so that thermal emission from the surface will be very weak. The magnetosphere, on the other hand, is the only part of the NS which will not disappear in the collapse as it is well outside the NS. According to the no-hair theorem, which prevents magnetic fields from puncturing the event horizon, the entire magnetic field should in principle detach and reconnect outside the horizon. This results in large currents and intense electromagnetic emission. Though the validity of the no-hair theorem in this context has been questioned by some (Lyutikov \& McKinney 2011), a strong magnetic shock wave moving at near the speed of light is indeed seen in 3D resistive MHD simulations of the collapse of non-rotating NSs (Dionysopoulou et al. 2013, Fig. 14).

The total power that can be radiated by the magnetosphere in the collapse is given by $P_{\mathrm{MS}}=\eta_{B}\left(B^{2} / 4 \pi\right) V / \Delta t$. Given that the

\footnotetext{
2 Indeed, it has been shown that the timescale for the collapse increases quadratically with the dimensionless spin (Baiotti et al. 2007).
}

magnetic field in the magnetosphere is decaying quickly with radius we here consider only a small shell comparable to the NS radius around the star, i.e. the volume is $V \simeq 4 \pi(2 R)^{3} / 3$. Moreover, $\eta_{B}$ is the fraction of magnetic energy that is available for dissipation and $\Delta t=1 \mathrm{~ms} t_{\mathrm{ms}}$ is the observed burst length. This is an upper limit, as the observed pulse widths could have been broadened by scattering.

Dionysopoulou et al. (2013) have also computed the temporal evolution of the ejected magnetic luminosity for the nonrotating case. Their Fig. 15 shows a dominant peak of order $\sim 0.1 \mathrm{~ms}$ width $\left(\sim 2 \times \tau_{\mathrm{ff}}\right)$ after the event horizon has formed, and a fainter precursor produced during the actual collapse. The dominant pulse is followed by additional pulses, which decay exponentially and signal the ringdown of the newly formed $\mathrm{BH}$. Precursor and the two leading pulses carry most of the transmitted power and contain about $5 \%$ of the available magnetic energy. Accordingly, we will use $\eta_{B}=0.05 \eta_{B, 5 \%}$ in the following. The bulk of the energy is released within $0.5 \mathrm{~ms}$.

The available power in the magnetosphere of a typical pulsar,

$$
P_{\mathrm{MS}} \simeq 4.2 \times 10^{43} \eta_{B, 5 \%} t_{\mathrm{ms}}^{-1} b_{12}^{2} r_{10}^{3} \mathrm{erg} \mathrm{s}^{-1} \text {, }
$$

is thus of the right order of magnitude compared to the observations (cf., Eq. (1)).

For simplicity we describe the magnetosphere with a simple aligned dipolar magnetic field $B$, rotating at $f \Omega_{\mathrm{K}}$ and filled with a pair plasma with particle number density $n_{\mathrm{e}}$, which we take to be a factor $\kappa_{\mathrm{GJ}}$ times the Goldreich-Julian density (Goldreich \& Julian 1969)

$n_{\mathrm{e}}=\kappa_{\mathrm{GJ}} \frac{B \Omega}{2 \pi c e} \simeq 1.8 \times 10^{13} f_{0.1} \kappa_{\mathrm{GJ}} b_{12} m_{2}^{1 / 2} r_{10}^{-3 / 2} \mathrm{~cm}^{-3}$.

In principle $\kappa_{\mathrm{GJ}}$ can have any value, but for our disucssion here we will typically assume that it is of order unity, $\kappa_{\mathrm{GJ}} \sim O(1)$.

In a standard pulsar magnetosphere electron and positrons $\left(\mathrm{e}^{+} / \mathrm{e}^{-}\right)$are spatially separated from each other in current sheets and glued to the magnetic field lines by the strong Lorentz forces. The strong magnetic shock waves generated in the collapse will accelerate the $\mathrm{e}^{+} / \mathrm{e}^{-}$pairs over spatial scales $\sim R$. Because the gyro radius of any of these particles is only $R_{\mathrm{gyr}} \simeq$ $1 \times 10^{-9} b_{12} \mathrm{~cm}$ - smaller than any radio wavelength, the particles will flow essentially along the magnetic field lines. Processes like synchrotron emission or self-absorption are therefore not applicable.

To describe the emission, we therefore use a basic relativistic curvature radiation model (Gunn \& Ostriker 1971; Ruderman \& Sutherland 1975) over radius $R$. This is, in fact, the typical scale of the distortions of the magnetic field lines induced by the collapse. Curvature radiation simply describes how relativistic electrons radiate when following a bent trajectory. Hence, this is an almost unavoidable emission process under these conditions.

The emitted power for a single relativistic electron or positron with Lorentz factor $\gamma$ is then $P_{\mathrm{e}}=2 \gamma^{4} e^{2} c / 3 R^{2}$, with a characteristic frequency

$v_{\text {curv }}=\frac{3 c \gamma^{3}}{4 \pi R} \simeq 7.2 \gamma^{3} r_{10}^{-1} \mathrm{kHz}$

When $\gamma \sim 1$, the corresponding wavelength is comparable to the size of the shock wave and the entire emission is coherent ${ }^{3}$,

3 The $N^{2}$ scaling of impulsive coherent emission mechanisms of charged particles in magnetic fields is in fact experimentally demonstrated by low-frequency radio emission observed from cosmic ray air shower fronts (Falcke \& Gorham 2003; Falcke et al. 2005), which has inspired our treatment. 
giving a total power of $P_{\mathrm{t}}=N_{\mathrm{e}}^{2} P_{\mathrm{e}}$, where $N_{\mathrm{e}}=n_{\mathrm{e}} V$ is the total number of particles. At higher frequencies and higher $\gamma$, the coherence length remains one wavelength but becomes smaller than the emitting region (e.g., Falcke \& Gorham 2003; Aloisio \& Blasi 2002). Hence, we have a number $N_{\text {slices }} \simeq$ $V R^{-2}\left(c / v_{\text {curv }}\right)^{-1}=\gamma^{3}$ of coherently emitting slices perpendicular to the line of sight (defined such that $N_{\text {slices }}=1$ for $\gamma=1$ ).

The emitted power then becomes $P_{\mathrm{t}}=\eta_{\mathrm{e}} N_{\text {slices }}^{-1} N_{\mathrm{e}}^{2} P_{\mathrm{e}}$, where $\eta_{\mathrm{e}}$ accounts for the fraction of $\mathrm{e}^{+} / \mathrm{e}^{-}$pairs that are accelerated to Lorentz factor $\gamma$. Hence,

$P_{\mathrm{t}} \simeq 7.0 \times 10^{43} \eta_{\mathrm{e}} \gamma f_{0.1}^{2} \kappa_{\mathrm{GJ}}^{2} b_{12}^{2} m_{2} r_{10} \mathrm{erg} \mathrm{s}^{-1}$.

Simple energy conservation imposes that $P_{\mathrm{MS}} \geq P_{\mathrm{t}}$ and it follows from Eqs. (4) and (7) that

$\gamma_{\max } \leq \frac{9 c \eta_{\mathrm{B}}}{8 R \Delta t \kappa_{\mathrm{GJ}}^{2} \eta_{\mathrm{e}} \Omega^{2}} \simeq 0.6 \eta_{\mathrm{e}}^{-1} \eta_{\mathrm{B}, 5 \%} f_{0.1}^{-2} t_{\mathrm{ms}}^{-1} \kappa_{\mathrm{GJ}}^{-2} r_{10}^{2} m_{2}^{-1}$,

where again $\Delta t=1 \mathrm{~ms} t_{\mathrm{ms}}$ is the duration of the pulse.

However, for the radio emission to propagate through the plasma, the radiation also has to be above the plasma frequency for a $\mathrm{e}^{+} / \mathrm{e}^{-}$pair plasma, $\omega_{\mathrm{p}}=\sqrt{4 \pi n_{\mathrm{e}} e^{2} / m_{\mathrm{e}}}$, which, for $n_{\mathrm{e}}=$ $n_{\mathrm{GJ}}\left(\kappa_{\mathrm{GJ}}\right)$, is

$\nu_{\mathrm{p}}=\frac{\omega_{\mathrm{p}}}{2 \pi}=\sqrt{\frac{e B \Omega}{2 \pi^{2} c m_{\mathrm{e}}}} \simeq 38.6 f_{0.1}^{1 / 2} \kappa_{\mathrm{GJ}}^{1 / 2} b_{12}^{1 / 2} m_{2}^{1 / 4} r_{10}^{-3 / 4} \mathrm{GHz}$.

For $\gamma \sim 1$ the ensuing $\mathrm{kHz}$ radio emission would be absorbed in the plasma and converted to plasma waves, which would again heat the $\mathrm{e}^{+} / \mathrm{e}^{-}$. Hence, in order for the radiation to escape effectively we need $v_{\text {curv }} \gtrsim v_{\mathrm{p}}$. Combining Eqs. (9) and (6), we find that in order to radiate the electrons need to have at least a Lorentz factor

$\gamma_{\min } \gtrsim 175.3 f_{0.1}^{1 / 6} \kappa_{\mathrm{GJ}}^{1 / 6} b_{12}^{1 / 6} m_{2}^{1 / 12} r_{10}^{1 / 12}$.

To reconcile Eqs. (8) and (10), and to avoid that more power is radiated than is available in the magnetosphere, we need to require that the fraction of relativistic electrons with $\gamma \geq \gamma_{\text {min }}$ should not be higher than

$\eta_{\mathrm{e}, \max } \lesssim 0.3 \% \eta_{\mathrm{B}, 5 \%} f_{0.1}^{-13 / 6} t_{\mathrm{ms}}^{-1} \kappa_{\mathrm{GJ}}^{-13 / 6} r_{10}^{23 / 12} b_{12}^{-1 / 6} m_{2}^{-13 / 12}$.

If we assume that the energy distribution of electrons in the shock front is a power-law of the form $\mathrm{d} N_{\mathrm{e}}(\gamma) / \mathrm{d} \gamma \propto \gamma^{-p}$, with $1<\gamma<\infty$, then $\eta_{\mathrm{e}, \max }=N\left(\gamma \geq \gamma_{\min }\right) / N(\gamma \geq 1)=\gamma_{\min }^{1-p}$. Hence, after requiring that $\gamma_{\min } \gtrsim 175$ (cf., Eq. (10)) and that $\eta_{\text {e, } \max } \lesssim 0.3 \%$ (cf., Eq. (11)), we conclude that $p \gtrsim 2.1$, which is not uncommon in astrophysical sources.

However, the very effective energy loss due to curvature radiation will ensure that the bulk of the $\mathrm{e}^{+} / \mathrm{e}^{-}$pairs also cannot have much higher Lorentz factors than in Eq. (10). Hence, the magnetosphere is already effectively dissipated by the $\mathrm{GHz}$ radio emission, which is bright enough to explain the observed FRBs.

This also naturally limits the maximum particle energy and suppresses additional X-ray or gamma-ray emission. Of course, higher Lorentz factors can still be reached by a smaller fraction of $\mathrm{e}^{+} / \mathrm{e}^{-}$pairs for which $\eta_{\mathrm{e}}(\gamma) \ll 1$ as long as they are not energetically dominant. Given that the available energy in the electrons is many orders of magnitude less than a typical GRB energy, this emission will not be detectable with current detectors.

We point out that the spectrum of curvature radiation is flat. However, given the rather complex nature of the shocked, exploding magnetosphere and the mixing between particles, plasma waves, and radio emission the resulting spectrum could be markedly different. This requires a more targeted effort, than what we can do here.

Before concluding, a couple of remarks. First, we have assumed spherical symmetry, so that beaming does not play a role in the energy budget. However, for a dipolar magnetic field the shock wave will have a bi-polar anisotropy and the emission could be beamed and increase the observed flux. Even for isotropic emission, the relativistic beaming will have the effect that the observer sees only a small patch of the emitting region. This, together with the ordered structure of the magnetosphere, could lead to significant polarization of the radiation. Moreover, absorption or scattering in the pulsar magnetosphere could modify the emission pattern. Obviously, radio emission can propagate unhampered along the polar axis but it could be scattered and absorped in a compact toroidal region as seen, e.g., in the double pulsar (Breton et al. 2012).

Second, our estimates assume $\kappa_{\mathrm{GJ}} \sim 1$ and $\eta_{B, 5 \%}=1$. The estimates of Lehner et al. (2012) in the case of rapidly rotating stars indicate that rotation in general increases the efficiency, but only up to $\eta_{B, 5 \%} \simeq 4$ (i.e. $\eta_{\mathrm{B}}=20 \%$ ). Hence, even when considering the extreme case of a very rapidly rotating pulsar, the order-of-magnitude estimate of the power in Eq. (4) is robust, with changes of at most a factor of four with rotation. The most constraining relation, however, is Eq. (11), which depends inversely on a high power of $\kappa_{\mathrm{GJ}}$ and which could in principle vary by many orders of magnitude in all directions for a generic model of FRBs. However, for $\kappa_{\mathrm{GJ}}<7 \%$ we would get $\eta_{\mathrm{e}, \max }>1$, given our scaling, and the model would break down. It is thus intriguing to note that - in logarithmic terms - the minimum density needed to establish a normal pulsar magnetosphere is not too far above the minimum density needed to explain FRBs.

\section{Summary and discussion}

We have argued that the short time scale of the observed FRBs, if indeed cosmological, may be indicative of a NS collapse. Moreover, the strong pulsar-like radio emission argues for emission associated with a magnetosphere and low baryon content. Supramassive rotating NSs can in principle provide such a setting.

If SURONs are formed, and there is no reason to believe they should not form, then they would collapse within several thousand to million years due to magnetic braking. The collapse of a SURON would proceed mostly quietly, producing a strong electromagnetic pulse due to the strong snapping of the magnetic field in the magnetosphere. Such a radio-emitting collapsar, which we dub "blitzar" due to its bright radio flash, could be a viable explanation for the recently discovered FRBs. The parameters needed for the NS are not significantly different from those of normal young pulsars, except for the higher mass. None of the processes we invoke in our scenario are in any way exotic. The SURON even need not be spinning very rapidly, as long as the mass excess is small.

Nonetheless, the energy demands to produce a FRB from a blitzar could quickly increase, if the observed radio spectrum is seen to extend with a flat spectrum to high frequencies or if the radiative efficiency of the magnetosphere is even lower than we assume. In this case, magnetic fields in excess of $10^{12} \mathrm{G}$ could be needed, which are in fact observed in the pulsar population.

Blitz $($ German $)=$ lightning flash. 
Formation scenarios for SURONs could involve a direct collapse in a $\mathrm{SN}$ explosion or spin-up due to accretion or merger with a white dwarf. It remains to be seen if any of those scenarios can produce magnetized SURONs at a sufficient rate and with high enough magnetic fields. The formation of very short-lived SURONs could have observational consequences in the form of an observed extended plateau phase in GRBs (Lipunova et al. 2009; Rowlinson et al. 2013). SURONs should also exist in our own Galaxy, but only as a small fraction of the pulsar population due to their short lifetime and the small fractional birthrate.

Future searches for FRBs can determine whether blitzars indeed trace the star formation rate in the universe and whether there is a connection to metallicity. This would lend support for an association with NS formation. Simultaneous optical and $\mathrm{X}$-ray data could constrain the emission process, baryon load, and the delay of the collapse.

Finally, we point out that the picture sketched here could provide an interesting window onto the formation of isolated stellarmass BHs, which would be otherwise invisible because the corresponding gravitational-wave emission is small. The ringdown of the event horizon could be visible in the radio emission of a blitzar as a succession of exponentially decaying sub-ms pulses. Detecting these decaying pulses will require observations with high signal-to-noise and low intergalactic and interstellar scattering, but would provide a unique signature for the birth of a $\mathrm{BH}$.

Acknowledgements. We thank A. Achterberg, S. Buitink, D. Champion, S. Johnston, J. Hessels, W. Hermsen, B. Gaensler, M. Kramer, P. Kumar, N. Langer, P. Mészáros, T. Oosting, P. Pizzochero, L. Stella, L. van Haaften, and F. Verbunt for discussions and useful suggestions. We also thank the first referee for timely comments and the second referee for constructive comments that have improved the manuscript. H.F. acknowledges support from an Advanced Grant of the European Research Council under the European Union's Seventh Framework Program (FP/2007-2013)/ERC Grant Agreement No. 227610. L.R. acknoweldges support from the DFG grant SFB/Transregio 7 and by "NewCompStar", COST Action MP1304.

\section{References}

Aloisio, R., \& Blasi, P. 2002, Astropart. Phys., 18, 183

Antoniadis, J., Freire, P. C. C., Wex, N., et al. 2013, Science, 340, 448

Baiotti, L., Hawke, I., Montero, P. J., et al. 2005, Phys. Rev. D, 71, 024035

Baiotti, L., Hawke, I., \& Rezzolla, L. 2007, Classical and Quantum Gravity, 24, 187

Baumgarte, T. W., \& Shapiro, S. L. 2003, ApJ, 585, 921

Bhattacharya, D., \& van den Heuvel, E. P. J. 1991, Phys. Rep., 203,

Bower, G. C., Whysong, D., Blair, S., et al. 2011, ApJ, 739, 76

Breton, R. P., Kaspi, V. M., McLaughlin, M. A., et al. 2012, ApJ, 747, 89

Burke-Spolaor, S., Bailes, M., Ekers, R., Macquart, J.-P., \& Crawford, III, F. 2011, ApJ, 727, 18

Cai, Y.-F., Sabancilar, E., Steer, D. A., \& Vachaspati, T. 2012, Phys. Rev. D, 86, 043521

Cavallo, G., \& Rees, M. J. 1978, MNRAS, 183, 359
Dahlen, T., Strolger, L.-G., Riess, A. G., et al. 2004, ApJ, 613, 189

de Mink, S. E., Langer, N., Izzard, R. G., Sana, H., \& de Koter, A. 2013, ApJ 764,166

Demorest, P. B., Pennucci, T., Ransom, S. M., Roberts, M. S. E., \& Hessels, J. W. T. 2010, Nature, 467, 1081

Dionysopoulou, K., Alic, D., Palenzuela, C., Rezzolla, L., \& Giacomazzo, B. 2013, Phys. Rev. D, 88, 044020

Duncan, R. C., \& Thompson, C. 1992, ApJ, 392, L9

Egorov, A. E., \& Postnov, K. A. 2009, Astron. Lett., 35, 241

Eichler, D., Livio, M., Piran, T., \& Schramm, D. N. 1989, Nature, 340, 126

Falcke, H., \& Gorham, P. 2003, Astropart. Phys., 19, 477

Falcke, H., Apel, W. D., Badea, A. F., et al. 2005, Nature, 435, 313

Fryer, C. L., \& Warren, M. S. 2004, ApJ, 601, 391

Gehrels, N., Sarazin, C. L., O'Brien, P. T., et al. 2005, Nature, 437, 851

Giacomazzo, B., Rezzolla, L., \& Stergioulas, N. 2011, Phys. Rev. D, 84, 024022

Goldreich, P., \& Julian, W. H. 1969, ApJ, 157, 869

Gunn, J. E., \& Ostriker, J. P. 1971, ApJ, 165, 523

Horiuchi, S., Beacom, J. F., Kochanek, C. S., et al. 2011, ApJ, 738, 154

Kashiyama, K., Ioka, K., \& Mszros, P. 2013, ApJ, 776, L39

Katz, J. I. 2013 [arXiv: 1309. 3538]

Kavic, M., Simonetti, J. H., Cutchin, S. E., Ellingson, S. W., \& Patterson, C. D. 2008, JCAP, 11, 17

Keane, E. F., Stappers, B. W., Kramer, M., \& Lyne, A. G. 2012, MNRAS, 425, L71

Langer, N. 1998, A\&A, 329, 551

Lee, W. H., \& Ramirez-Ruiz, E. 2007, New J. Phys., 9, 17

Lehner, L., Palenzuela, C., Liebling, S. L., Thompson, C., \& Hanna, C. 2012, Phys. Rev. D, 86, 104035

Lipunova, G. V., Gorbovskoy, E. S., Bogomazov, A. I., \& Lipunov, V. M. 2009, MNRAS, 397, 1695

Loeb, A., Shvartzvald, Y., \& Maoz, D. 2014, MNRAS, in press [arXiv: 1310.2419]

Lorimer, D. R., Bailes, M., McLaughlin, M. A., Narkevic, D. J., \& Crawford, F. 2007, Science, 318, 777

Lorimer, D. R., Karastergiou, A., McLaughlin, M. A., \& Johnston, S. 2013, MNRAS, 436, L5

Luan, J. 2014 [arXiv: 1401.1795]

Lyutikov, M. 2013, ApJ, 768, 63

Lyutikov, M., \& McKinney, J. C. 2011, Phys. Rev. D, 84, 084019

Nakar, E. 2007, Phys. Rep., 442, 166

Paczynski, B. 1986, ApJ, 308, L43

Page, D., Prakash, M., Lattimer, J. M., \& Steiner, A. W. 2011, Phys. Rev. Lett., 106, 081101

Pshirkov, M. S., \& Postnov, K. A. 2010, Ap\&SS, 330, 13

Rowlinson, A., O'Brien, P. T., Metzger, B., Tanvir, N., \& Levan, A. 2013, MNRAS, 430, 1061

Ruderman, M. A., \& Sutherland, P. G. 1975, ApJ, 196, 51

Siemion, A. P. V., Bower, G. C., Foster, G., et al. 2012, ApJ, 744, 109

Somov, B. V. 2011, Astron. Rep., 55, 962

Takami, K., Rezzolla, L., \& Yoshida, S. 2011, MNRAS, 416, L1

Tauris, T. M., Langer, N., \& Kramer, M. 2011, MNRAS, 416, 2130

Thornton, D., Stappers, B., Bailes, M., et al. 2013, Science, 341, 53

Totani, T. 2013, PASJ, 65, L12

van den Heuvel, E. P. J., \& Bonsema, P. T. J. 1984, A\&A, 139, L16

van Haaften, L. M., Nelemans, G., Voss, R., et al. 2013, A\&A, 552, A69

Vietri, M., \& Stella, L. 1998, ApJ, 507, L45

Woosley, S. E. 1993, ApJ, 405, 273

Woosley, S. E., \& Bloom, J. S. 2006, ARA\&A, 44, 507

Yoon, S.-C., Langer, N., \& Norman, C. 2006, A\&A, 460, 199 